\title{
Application of long-period-grating sensors to respiratory plethysmography
}

\author{
Thomas Allsop \\ Karen Carroll \\ Glynn Lloyd \\ David J. Webb \\ Aston University \\ Photonics Research Group \\ Aston Triangle \\ Birmingham B4 7ET \\ United Kingdom
}

\author{
Martin Miller \\ University Hospital Birmingham NHS Trust \\ Department of Medicine \\ Birmingham B29 6JD \\ United Kingdom
}

\section{Ian Bennion}

Photonics Research Group

Aston Triangle

Birmingham B4 7ET

United Kingdom

\begin{abstract}
A series of in-line curvature sensors on a garment are used to monitor the thoracic and abdominal movements of a human during respiration. These results are used to obtain volumetric tidal changes of the human torso in agreement with a spirometer used simultaneously at the mouth. The curvature sensors are based on long-period gratings (LPGs) written in a progressive three-layered fiber to render the LPGs insensitive to the refractive index external to the fiber. A curvature sensor consists of the fiber long-period grating laid on a carbon fiber ribbon, which is then encapsulated in a low-temperature curing silicone rubber. The sensors have a spectral sensitivity to curvature, $d \lambda / d R$ from $\sim 7-\mathrm{nm} \mathrm{m}$ to $\sim 9-\mathrm{nm} \mathrm{m}$. The interrogation technique is borrowed from derivative spectroscopy and monitors the changes in the transmission spectral profile of the LPG's attenuation band due to curvature. The multiplexing of the sensors is achieved by spectrally matching a series of distributed feedback (DFB) lasers to the LPGs. The versatility of this sensing garment is confirmed by it being used on six other human subjects covering a wide range of body mass indices. Just six fully functional sensors are required to obtain a volumetric error of around 6\%. () 2007 Society of Photo-Optical Instrumentation Engineers. [DOI: 10.1117/1.2821198]
\end{abstract}

Keywords: curvature sensing; long-period gratings; respiratory monitoring.

Paper 06382R received Dec. 22, 2006; revised manuscript received Jul. 24, 2007; accepted for publication Jul. 31, 2007; published online Dec. 19, 2007.

\section{Introduction}

Noninvasive measurement of thoracoabdominal surface motion can provide a comprehensive appreciation of respiratory function and enhance our understanding of respiratory physiology. Knowledge of the total and regional deflection of the surface during breathing not only provides a key to the volumetric flow of gases, but also provides insight to the thoracic and abdominal contributions and the recruitment of muscles. Until now, research in this field has been predominantly laboratory based; however, the provision of a reliable quantitative clinical tool used for making ventilatory measurements in ambulatory patients would advance routine respiratory function monitoring to a point similar to that accomplished with 24-h cardiovascular monitoring in recent years. Surface sensing of ventilation from the chest and abdomen would allow real-time ventilatory monitoring of subjects in difficult environments, such as critical care wards in hospitals or during occupational exposures to noxious agents. In this way, this methodology could facilitate the diagnosis and management of several pulmonary conditions such as occupational asthma, for example.

Konno et al. ${ }^{1}$ first demonstrated that respiratory gas flow could be measured at the surface of the chest and abdomen, thus negating the need for measurement of flow at the mouth, which is the clinical standard at present. A number of mea-

Address all correspondence to Thomas Allop, Aston Univ., Aston TriangleBirmingham, West Midlands B4 7ET, United Kingdom; Tel: 44(0)121-2043484; Fax: 44(0)121-359-0156; E-mail: t.d.p.allsop@aston.ac.uk surement devices have been developed following Konno et al. The paradigms include: respiratory inductive plethysmography (RIP), ${ }^{2}$ optical reflectance plethysmography (ORP) ${ }^{3}$ and the use of magnetometers. ${ }^{4}$ Each of these has certain limitations for use in ambulatory respiration monitoring. These pertain to the accuracy over wide variations in tidal volume and changes in posture after the calibration of RIP. ${ }^{5}$ This can be addressed by using a sensor array; ${ }^{6}$ however, the solution relies heavily on model-based signal processing. The cost and realization of a sufficiently dense array of magnetometers is prohibitive. While the ORP methods lack such limitations, they rely on an off-body reference with which to track specified anatomical locations. Present instrumentation is bulky and generally has to be operated while the patient occupies a confined area. It follows that the technique is not suitable for routine ambulatory monitoring.

The fundamental requirement is to create a highly compliant sensor with which to track selected anatomical positions on the chest and abdomen surface. A possible solution to this problem is to use fiber long-period grating (LPG) as the sensing element. Recent work $^{7-10}$ using fiber LPGs to monitor shape changes (bending) is promising, but there is one major disadvantage: to interrogate LPGs, expensive broadband light sources and optical spectrum analysers (OSAs) are usually required.

1083-3668/2007/12(6)/064003/13/\$25.00 @ 2007 SPIE 


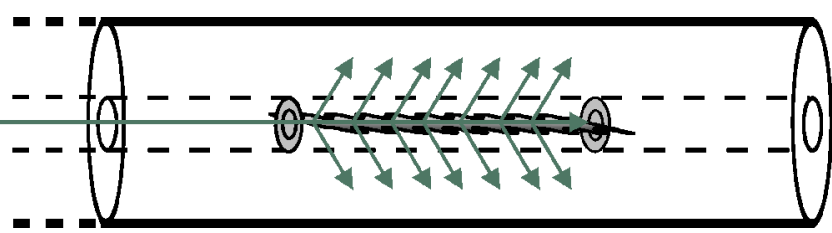

Fig. 1 Optical fiber long-period grating.

This work describes research investigating a possible solution to this problem involving an array of LPG curvature sensors interrogated by a set of distributed feedback lasers. First, it is shown that this curvature sensor is suitable for the application of respiratory plethysmography. Second, the curvature sensors are adhered to a garment to enable them to be used to detect the shape variations of the chest and abdominal regions of a human torso during respiration using the transmission spectrum of the LPGs' attenuation bands. Third, a curvature sensor array is used in conjunction with a signal-processing scheme based on derivative spectroscopy ${ }^{11}$ to show the multiplexing capability of the interrogation scheme and to demonstrate the monitoring of a human subject during respiration. Fourth, analysis is performed on the acquired data to show that data from only six fully functional sensors is required to obtain approximately $6 \%$ volumetric error for the human subject used. Finally, the curvature sensing garment is used to monitor a variety of human subjects with body mass indices from 18 to 32. To the best of the authors' knowledge, this is the first application of a multiplexed LPG array.

Potentially, this sensing scheme can be both relatively inexpensive to implement and compact in size, meaning that the entire system could be attached to the patient being monitored.

\section{Curvature Sensor}

The curvature sensor utilizes an LPG as the sensing element. LPGs are photoinduced fiber devices that couple light from the core of a single-mode optical fiber into the cladding at discrete wavelengths, thereby generating attenuation bands in the transmission spectrum of the optical fiber core (see Fig. 1).

Over the last few years, the fiber LPG has found numerous applications in the field of sensing. This is because the LPGs are sensitive to strain $(\varepsilon)$, temperature $(T)$, and the surrounding refractive index $\left(n_{s}\right) .^{10,12}$ Most relevant to the current work, LPGs have also been used as curvature sensors, ${ }^{13,14}$ since the LPG's attenuation bands are spectrally sensitive to bending, which induces both a wavelength shift and a change in the spectral profile of the attenuation band. The wavelength shift of the attenuation band arises from the phase match condition of the LPG, given by ${ }^{9}$

$$
\begin{aligned}
\lambda_{i}= & \delta n_{\mathrm{eff}} \Lambda \equiv\left[n_{\mathrm{eff}_{\text {core }}}\left(\lambda_{\iota}, n_{1}, n_{2}, T, \mu \varepsilon, \boldsymbol{R}\right)\right. \\
& \left.-n_{\mathrm{eff}_{\text {cladding }}^{\nu}}\left(\lambda_{i}, n_{1}, n_{2}, T, \mu \varepsilon, n_{s}, \boldsymbol{R}\right)\right] \Lambda,
\end{aligned}
$$

where $n_{\text {eff }}$ is is the effective index of the core mode and $n_{\text {eff }}^{\nu} \quad$ is the effective index of the $\nu$ 'th radial cladding mode, both indices being also dependent on the core and cladding refractive indices and wavelength $\lambda . \Lambda$ is the period of

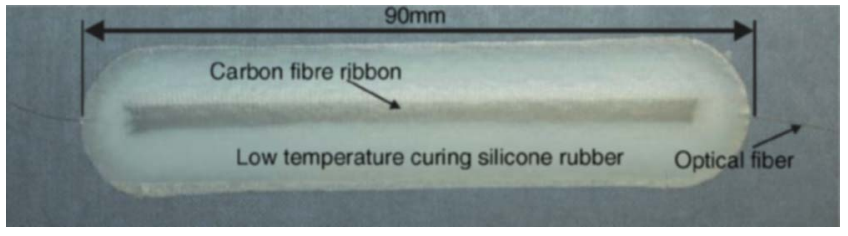

Fig. 2 The curvature sensor.

the grating. While Eq. (1) gives the spectral position of the attenuation band, the magnitude of the spectral shift induced by the measurands $\left(\varepsilon, T, n_{s}\right)$ is dependent on two factors: the difference between the effective refractive indices of the core and cladding modes, and also the difference between the group effective refractive indices of the two modes. ${ }^{14}$ The spectral sensitivity of LPGs to bending arises from two major components: the varying strain across the fiber and the effective change in the refractive index profile of the fiber induced by the bend itself. These can be visualized by the conformal mapping technique. ${ }^{15}$ The bending of the optical fiber induces changes to the propagation constants of the cladding modes, thus changing the group refractive indices as well as the effective refractive indices.

The main component of the curvature sensor is the LPG with a length of about $7 \mathrm{~cm}$ that is written in a progressive three-layered fiber using the point by point method. ${ }^{16}$ The result of using such a fiber is that the LPG produces attenuation bands that are insensitive to changes in the surrounding medium's refractive index. The LPG is laid on a carbon fiber ribbon, the arrangement then being encapsulated in a lowtemperature $\left(90^{\circ} \mathrm{C}\right)$ curing silicone rubber. This type of silicone rubber is used to prevent significant changes to the LPG's attenuation bands due to thermal annealing (see Fig. 2 ). The use of the progressive three-layered fiber prevents there from being any shift in the LPG's attenuation bands when it is embedded in the rubber.

The sensor construct was designed to prevent the fiber from experiencing significant axial strain while at the same time providing a flexible stage for bending as well as a thermally insulating layer, reducing the effects of rapid ambient temperature fluctuations. The thermal spectral sensitivity results are illustrated in Fig. 3, along with the normal temperature variation of the skin. ${ }^{17}$ The sensor is intended to be worn close to the skin and be insulated from the surrounding air, and so this is the temperature range that the sensor must normally accommodate.

Measurements were preformed on the attenuation band associated with $\mathrm{HE}_{1,3}$ cladding mode at a wavelength of $1510 \mathrm{~nm}$. The temperature sensitivity was measured by immersing the sensor in a bath of hot water and monitoring the wavelength shift as the water cooled. This approach gave a sensitivity of $d \lambda / d T=0.0964 \pm 0.5 \times 10^{-3} \mathrm{~nm}^{\circ} \mathrm{C}^{-1}$. It should be noted, however, that this way of determining the temperature sensitivity may not necessarily correspond all that well to the way in which the sensor will be used in practice. The sensing fiber is embedded inside an insulating material and in the intended application; while one side of this material is exposed to the vagaries of the environment, the other side will be closer to body temperature, reducing the temperature variation experienced by the grating. It is worth noting that the 


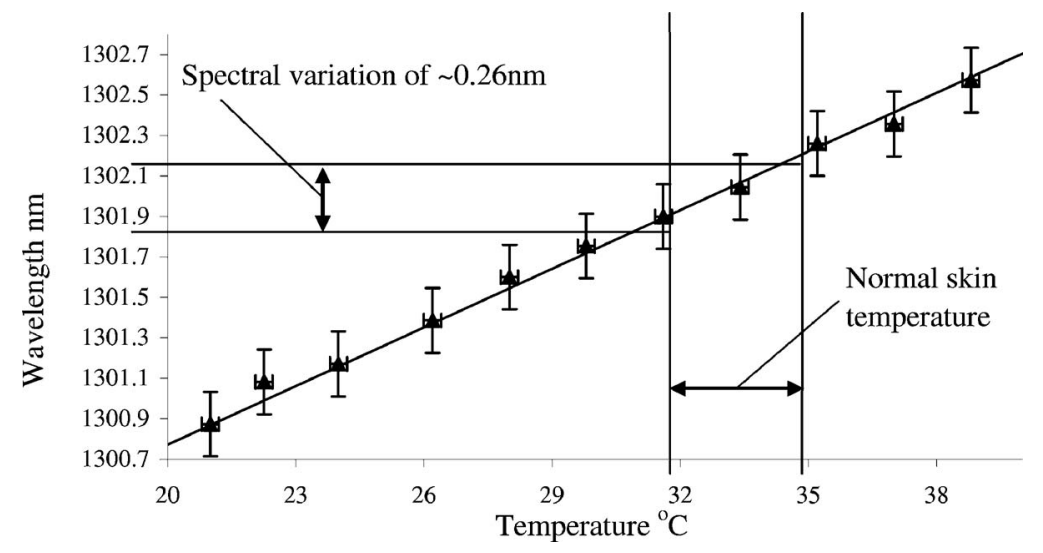

Fig. 3 The spectral sensitivity of a curvature sensor (LPG period $=350 \times 10^{-6} \mathrm{~m}$ ). The error bars indicate the accuracy of the OSA $( \pm 0.04 \mathrm{~nm}$ ).

temperature sensitivity of long-period gratings is dependent on the difference in thermo-optic coefficients of the materials used for core and cladding glass ${ }^{9}$ these can, in principle, be controlled to make the LPGs insensitive to temperature variations.

The second investigation was to determine the influence of transverse compression on the sensor. This was achieved by placing flat weights on the top curved surface of the sensor, which was itself placed on an optical bench. The experimental results are shown in Fig. 4. The compression load applied to the sensor ranged up to $23 \mathrm{~N}$ (much higher than any likely force in the intended application). There was a small variation of the central wavelength of the attenuation band observed ( $\sim \pm 0.3 \mathrm{~nm}$ ), but there was no general trend observed as a function of compression. The wavelength variation is probably due to a lack of reproducibility in the positioning of the load and small temperature variations. The error bars shown in Fig. 4 again indicate the accuracy of the OSA used.

The effect of axial strain on the spectral characteristics of a curvature sensor was also investigated. This was done by clamping the curvature sensor between two towers; one of the clamps was mounted on a translation stage, which was moved outward to induce an axial strain in the sensor. The sensor was held in two ways, first by clamping only the rubber section of the sensor, and second by clamping to both the rubber and carbon fiber sections of the sensor (see Fig. 5). The axial strain sensitivities for both clamping arrangements were found to be similar, being $5.95 \times 10^{-5} \mathrm{~nm} \mu \varepsilon^{-1}$ for the clamping of both rubber and carbon, and 5.98

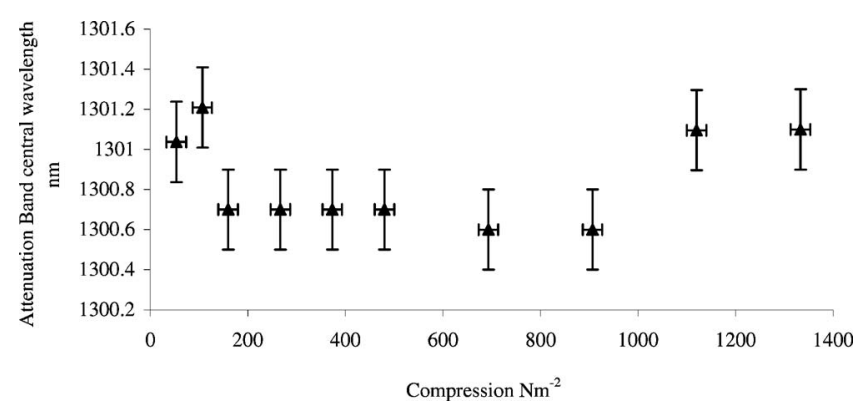

Fig. 4 Spectral stability of the curvature sensor with a sensing element under compression (LPG period $=350 \times 10^{-6} \mathrm{~m}$ ). $\times 10^{-5} \mathrm{~nm} \mu \varepsilon^{-1}$ for clamping just the rubber. These figures can be compared favorably with the strain sensitivity of the bare fiber, $3.95 \times 10^{-4} \mathrm{~nm} \mu \varepsilon^{-1}$. Thus the carbon fiber may have reduced considerably the sensitivity to axial strain of the packaged sensor.

\section{Interrogation Schemes}

For the work described, three different methods for interrogating the LPGs have been implemented. Basic testing was carried out using an OSA for direct monitoring of the wavelength of the center of the attenuation band. Such an approach is convenient in the laboratory but undesirable in a practical system due to reasons of cost, size, and low data rate. Consequently, two variant interrogation schemes based on derivative spectroscopy have been developed, one involving analog (continuous) modulation of the optical source - a distributed feedback (DFB) laser — and the other digital (discrete) modulation.

Derivative spectroscopy was originally developed for wavelength modulation absorption spectrometry, and usually involves applying a small sinusoidal wavelength modulation to the optical source wavelength. ${ }^{18}$ This technique is mostly used for environmental monitoring and remote sensing, as well as spectrochemical applications, e.g., $\mathrm{NO}_{2}$ and $\mathrm{CCL}_{4}$ detection.

We have implemented this approach to detect the changes in the spectral transmission profile of the LPG's stop band induced by the bending of the LPG. Modulating the DFB laser wavelength at a given frequency will generate a series of harmonics at the modulating frequency ${ }^{19}$ in the output signal transmitted by the LPG. It has been shown that the in-phase component of the $n$ 'th harmonic output is proportional to the $n$ 'th derivative of the spectral profile under investigation. ${ }^{18}$ The amplitudes of the first and second harmonics are therefore proportional to the first and second derivatives of the spectral transmission function of the sensor, and the utility of our approach relies on the ratio of those derivatives being a unique function of the position in the spectral profile, as well as being independent of any attenuation in the system. We have previously reported this interrogation technique in a system where a single LPG was interrogated using a pigtailed DFB laser as the light source. ${ }^{19}$ 


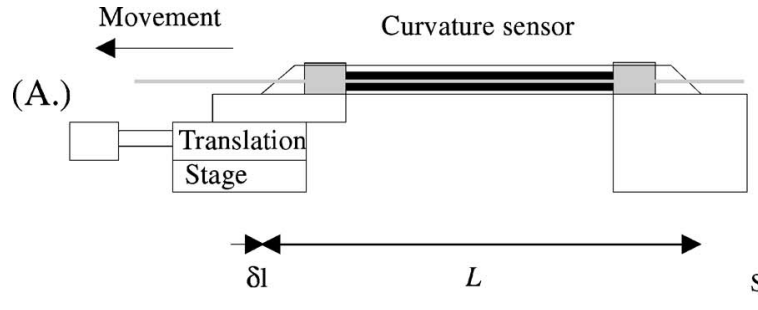

Silicone rubber

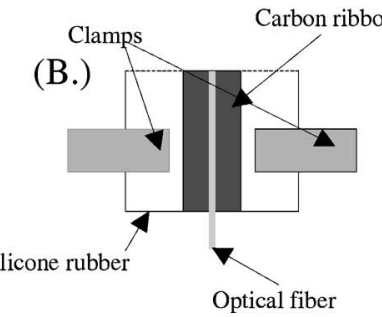

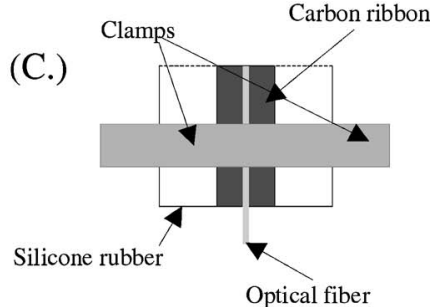

Fig. 5 (a) Schematic of the axial strain arrangement. (b) Clamping onto rubber. (c) Clamping onto the carbon fiber strip.

We refer to the prior technique as analog derivative spectroscopy. For our latest work, a digital variant on this approach was also employed, where the DFB laser wavelength was cycled through three discrete values, with the transmitted intensity being recorded each time. The derivatives of the spectral profile are then approximated using these difference values (see Fig. 6). This digital derivative spectroscopy approach was more convenient to interface to the microcontroller-based signal processing system developed for this project. In the final system, two DFB lasers were used to address a total of up to ten curvature sensors.

\section{Characterization of Curvature Sensors}

A calibration of the curvature sensors was first performed, the bending of the LPG sensor being achieved using the experimental setup shown in Fig. 7.

The LPG sensor was clamped between two towers; one of the clamps was mounted on a translation stage, which was moved inward to induce a bend in the optical fiber. For this arrangement, where the LPG is midway between the clamps, Ref. 20 gives the sensor's curvature $R$ as:

$$
R=\frac{2 \cdot d}{\left(d^{2}+L^{2}\right)},
$$

where $L$ is the half distance between the edges of the two towers and $d$ is the bending displacement at the center of the LPG.
First, the central wavelengths of the attenuation bands were monitored with an OSA as a function of curvature to assess the performance of the curvature sensor in terms of curvature range and sensitivity. An example of the spectral sensitivity of a sensor (period $=532 \times 10^{-6} \mathrm{~m}$, length $=6 \mathrm{~cm}$ ) is shown in Fig. 8(a). Having obtained this information, the digital derivative spectroscopy interrogation technique could be implemented and optimized, by adjusting the central wavelength of the DFB laser and the size of the wavelength steps. Figure 8 (b) illustrates, using a parametric plot, the evolution of the amplitudes of the first and second harmonics as the curvature is varied over the range 0 to $4.2 \mathrm{~m}^{-1}$, while Fig. 8 (c) is the calibration curve showing the output from the system (obtained by taking the arctangent of the ratio of the first and second harmonic amplitudes) as a function of sensor curvature.

Consideration needs to be given to the sensitivity of the sensors to other environmental parameters, the main one being temperature. Using the OSA to interrogate a curvature sensor, a spectral sensitivity of $6.87 \pm 0.11 \mathrm{~nm} \mathrm{~m}$ was found in approximately the linear response region. Modeling of the complete spectral response of the curvature sensor using a sixth order polynomial [see Fig. 8(a)] results in a curvature error (rms deviation from the polynomial) of $\pm 0.035 \mathrm{~m}^{-1}$. Taking into account the normal bodily temperature variation of $3^{\circ} \mathrm{C}$ leads to an overall error in curvature of $\pm 0.068 \mathrm{~m}^{-1}$. Implementing the digital derivative spectroscopy interrogation technique introduces an error of \pm 0.062 , obtained by fit-

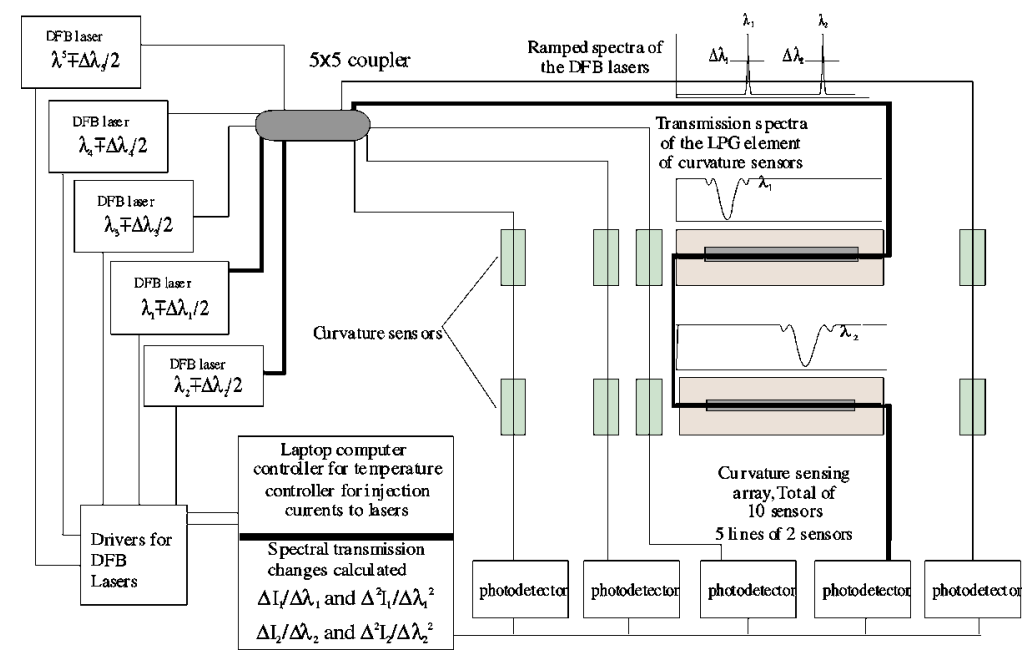

Fig. 6 A schematic of the interrogation/multiplexing scheme. 


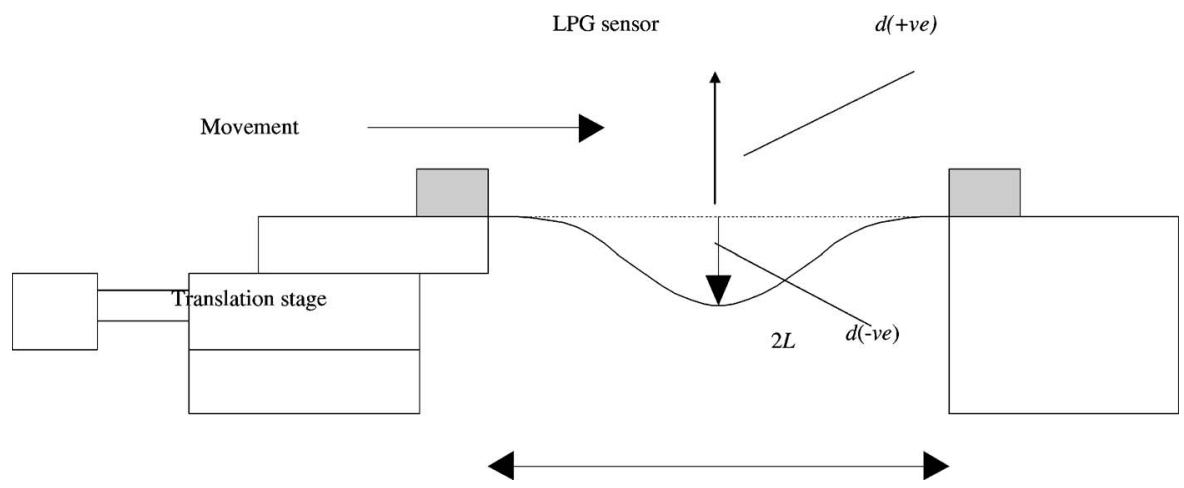

Fig. 7 Schematic of bending apparatus.

ting a sixth order polynomial to the inverse tangent of the ratio of the first and second derivatives, which translates to a similar curvature error of $\pm 0.067 \mathrm{~m}^{-1}$. When normal bodily temperature variations are taken into account, this yields a total error in curvature of $\pm 0.086 \mathrm{~m}^{-1}$.

The major environmental parameter that will affect this curvature sensing scheme is the intrinsic temperature sensitivity of the LPG. This can, in principle, be overcome by using a discrimination scheme such as that described in Ref. 21, but to assess the validity of such an approach, it is necessary to characterize the temperature-curvature cross-sensitivity. To do this, a similar procedure was adopted to the one described in Ref. 22. Using a Taylor expansion for the temperature and bending-induced change in wavelength, and also assuming that there is a linear relationship between wavelength shift of the attenuation band and both curvature $(R)$ and temperature $(T)$, we have the following expression, which contains the cross-sensitivity in the final term on the right hand side:

$$
\Delta \lambda=\delta n_{\mathrm{eff}_{\nu}} \Lambda+\frac{\partial \lambda}{\partial T} \cdot \Delta T+\frac{\partial \lambda}{\partial R} \cdot \Delta R+\frac{\partial^{2} \lambda}{\partial R \partial T} \cdot \Delta T \Delta R
$$

Using the expression given in Ref. 10 for $(d \lambda / d T)$ and differentiating with respect to curvature $R$ yields the following expression for the spectral cross sensitivity,

$$
\begin{aligned}
\frac{\partial^{2} \lambda}{\partial T \partial R}= & \frac{-\Delta \xi \cdot \lambda}{\left(\delta n_{\mathrm{eff}}-\delta n_{g}\right)^{2}} \cdot\left[\frac{\partial \delta n_{\mathrm{eff}}}{\partial R}-\frac{\partial \delta n_{g}}{\partial R}\right] \\
& +\Lambda \alpha \cdot\left[2 \frac{\delta n_{\mathrm{eff}}}{\delta n_{g}} \cdot \frac{\partial \delta n_{\mathrm{eff}}}{\partial R}-\left(\frac{\delta n_{\mathrm{eff}}}{\delta n_{g}}\right)^{2} \cdot \frac{\partial \delta n_{g}}{\partial R}\right] \\
& +\alpha \cdot \frac{\left(\delta n_{\mathrm{eff}}\right)^{2}}{\delta n_{g}} \cdot \frac{\partial \Lambda}{\partial R} .
\end{aligned}
$$

The undefined terms in this expression are $\alpha$, the thermal expansion coefficient of the core, and $\Delta \xi \nu$, the differential thermo-optic coefficient between the core and the cladding material, given in Ref. 23. Using the results obtained from a 2-D curvilinear hybrid mode eigenvalue equation and the condition that the amount of birefringence induced in a typical single-mode fiber is negligibly small for curvatures (pure bending) of $<2 \mathrm{~m}^{-1},{ }^{24}$ (i.e., there is no splitting of the attenuation band), the spectral cross-sensitivity between temperature and curvature was calculated to be $3 \times 10^{-3} \mathrm{~nm} \mathrm{~m}^{\circ} \mathrm{C}^{-1}$. This figure is small. Over the experimental measurement range, it gives a relative cross-sensitivity error of $0.08 \%$ of full scale curvature $/{ }^{\circ} \mathrm{C}$, which is completely negligible.
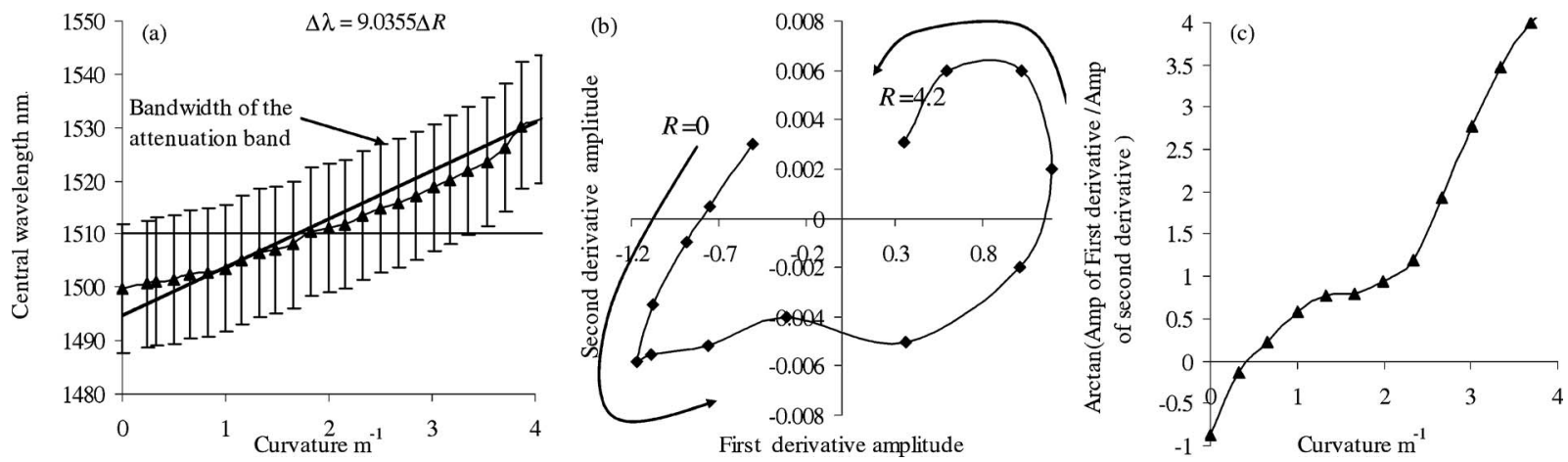

Fig. 8 An example of the calibration procedure using the digital derivative spectroscopy interrogation technique. (a) The spectral response of the sensor measured using an optical spectrum analyzer to check the usable curvature range. (b) Parametric plot of the first and second derivatives as a function of curvature. (c) Optimizing the output response of the sensing scheme as a function of curvature, to obtain the best linearity with maximized sensitivity. 

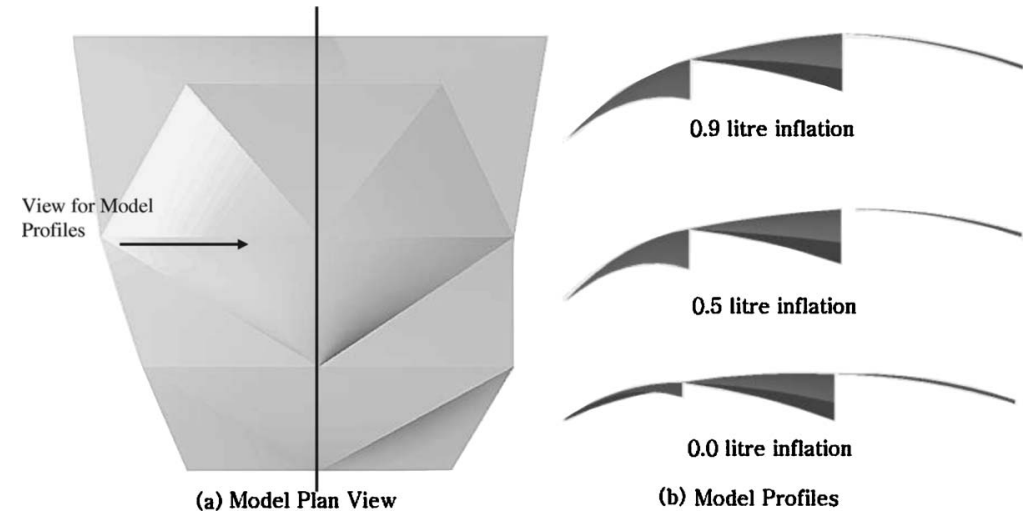

Fig. 9 Shape reconstruction of a resuscitation training manikin using the derivative spectroscopy interrogation scheme with the curvature sensors at 13 locations: (a) shows the overall reconstruction of the manikin, and (b) shows a side view of the profile of the manikin at various inflations.

\section{Monitoring Simulated Respiration Using a Curvature Sensor Array}

First, to validate the sensor design and the use of the derivative spectroscopy interrogation technique for the intended application, a commercial manikin used as a resuscitation training aid is employed. ${ }^{25}$ This comprises a rigid underframe, over which is stretched a polymer skin. An air bag placed between the frame and skin, when inflated or deflated, is then used to simulate expansion and contraction of the surface of the torso in similar volumetric proportions to that of breathing. This provides a test platform of similar dimensions and shape variation to those that are expected in use, but with easily controllable and highly reproducible shape changes.

Two in-line curvature sensors were used to record the variation of the curvature at various states of inflation from 0 to $0.9 \mathrm{~L}$ in increments of $0.3 \mathrm{~L}$. Two locations were simultaneously monitored over the volume range, with the sensors then being moved to different locations and the manikin inflated using an Ambubag over the same volume range. The curvature was measured in two ways, first by monitoring the wavelength shift of the transmission spectrum obtained from an OSA, and second using the derivative spectroscopy interrogation technique described earlier. It was found that the sensing scheme was readily able to detect the variations in the curvature. Examples can be seen in Fig. 9, which depicts the static reconstruction of surface curvature from the manikin using commercial computer-aided design software (SolidWorks, Concord, MA). ${ }^{26}$ In this case, the overall profile is determined by successively linking the arcs of curvature in their respective planes. Figure 9(a) shows the location of sensing sites on the manikin at the edges of the triangular patches, giving 13 sensors (two on the top edge). Smoothing of the profile is accomplished by setting peripheral relationships between curves and the application of lofting algorithms built into the software. Of course, the relatively small number of sensing locations employed in this experiment reduces the fidelity of the model. Figure 9 shows that the sensors are sensitive enough to distinguish between various inflation volumes, as shown in the lateral profiles in Fig. 9(b).

\section{Monitoring Human Respiration Using a Curvature Sensor Array}

A preliminary experiment was conducted to confirm the suitability of the curvature sensors to the application of human plethysmography. This was achieved with four curvature sensors (length of $15 \mathrm{~cm}$ ) in an in-line (series) configuration. The sensor array was stitched to a Lycra band for a practical evaluation of the sensors. All the curvature sensors used for this evaluation had similar spectral sensitivities (ranging from about $7 \mathrm{~nm} \mathrm{~m}$ to about $9 \mathrm{~nm} \mathrm{~m}$ ) with a curvature resolution of $3.5 \times 10^{-2} \mathrm{~m}^{-1}$.

The Lycra vest was placed on a human male torso in two different orientations; first so that the array covered the front of the torso (rectus abdominus/sternal pectorallis major region), and second with the array along the side of the torso, under the arm (internal/external oblique region). The sensors were illuminated with a broadband light source, and the wavelength shifts of the attenuation bands were monitored with an OSA controlled by a computer (see Fig. 10). The capture rate was limited to about $0.5 \mathrm{~Hz}$ due to the delay in transferring data from the OSA to the computer.

The sensor array monitored the thorax movement of a subject taking a long tidal breath. An example is shown in Fig. 11(a) of the curvature variation of one sensor located on the front of the torso. As a control, with the subject holding their breath, several measurements were recorded and the noise in the system - as represented by their deviations - was found to be the same as obtained in the initial characterization of the sensors. Thus no additional error had been introduced by mounting the sensors on the vest.

Inspecting Fig. 11(a) and the responses of the other curvature sensors revealed variations in curvature for different long tidal breaths, but this is expected due to the fact that we were monitoring a human rather than the manikin, the movements of which are far more reproducible. The important point is that the general behavior is the same during each breath, and this was found for all the sensors at the various locations on the thorax.

While the sensors were being monitored, the exhaled volumes were simultaneously recorded at the mouth of the sub- 


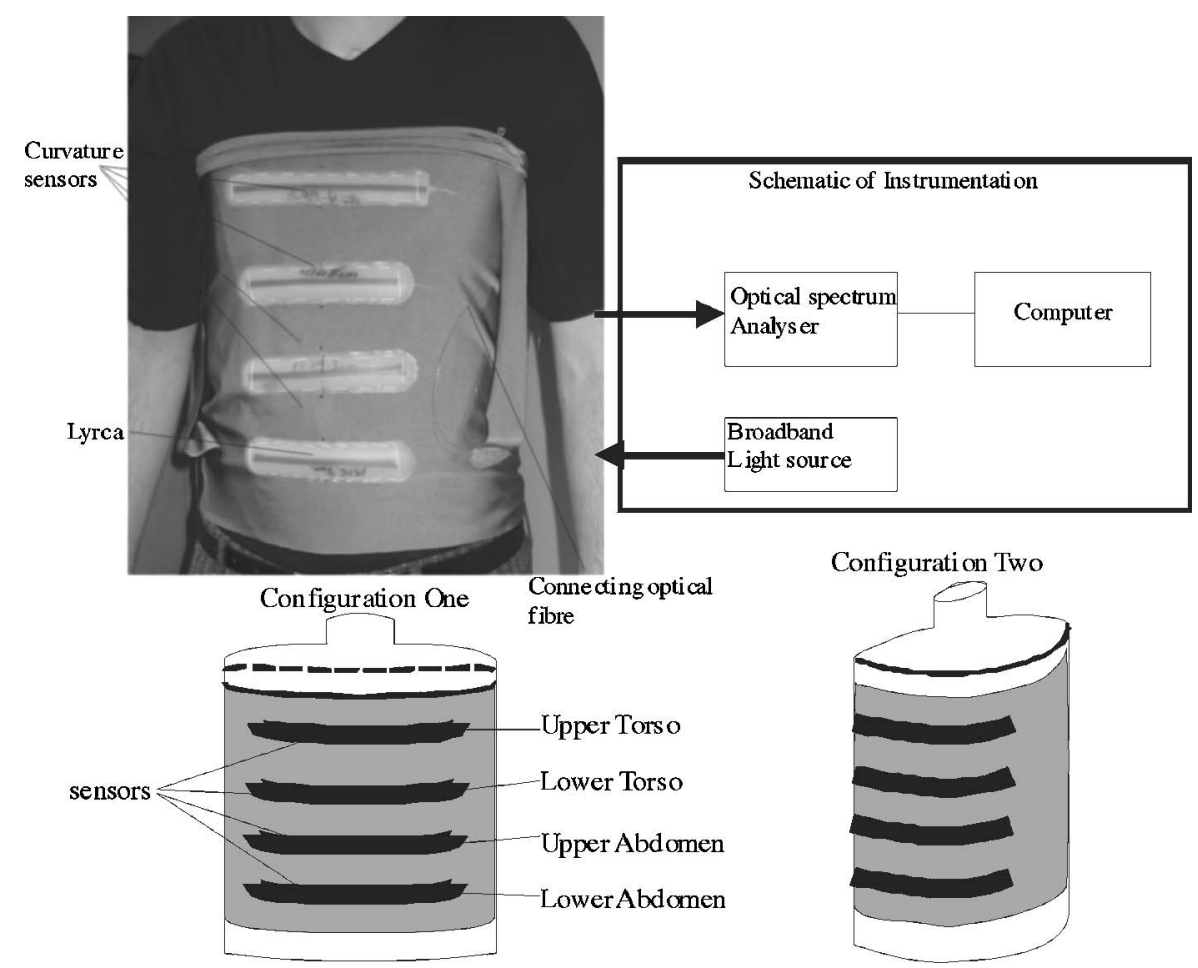

Fig. 10 Schematic of optical instrumentation used for the monitoring of the curvature sensors.

ject using a turbine transduction spirometer system (Micro Medical, United Kingdom). Linear regression was applied to find the relationship between the volumes measured with the spirometer and the measured curvatures to predict exhaled volume from the sensor signals for the two spatial locations of the sensor array. It can be shown that from a simple system of these sensors, an accurate assessment of tidal breathing can be made, that is similar in accuracy to that previously found for an external optical system using 80 sensor points on the thorax. ${ }^{27}$ Figure 11(b) shows an example of a direct comparison of the volume measured from the mouth with a spirometer and the volume calculated from the curvature-sensing array using the relationship obtained from linear regression.

While this comparison showed encouraging agreement, the data rate, limited by the capture time of data from the OSA, was much too low to be of practical use. Consequently, at this point the digital derivative spectroscopy technique was implemented (see Sec. 3). The system was designed to allow for up to 25 sensors to be addressed using five sources and five detectors, though in practice only nine sensors were used, along with two sources and five detectors.
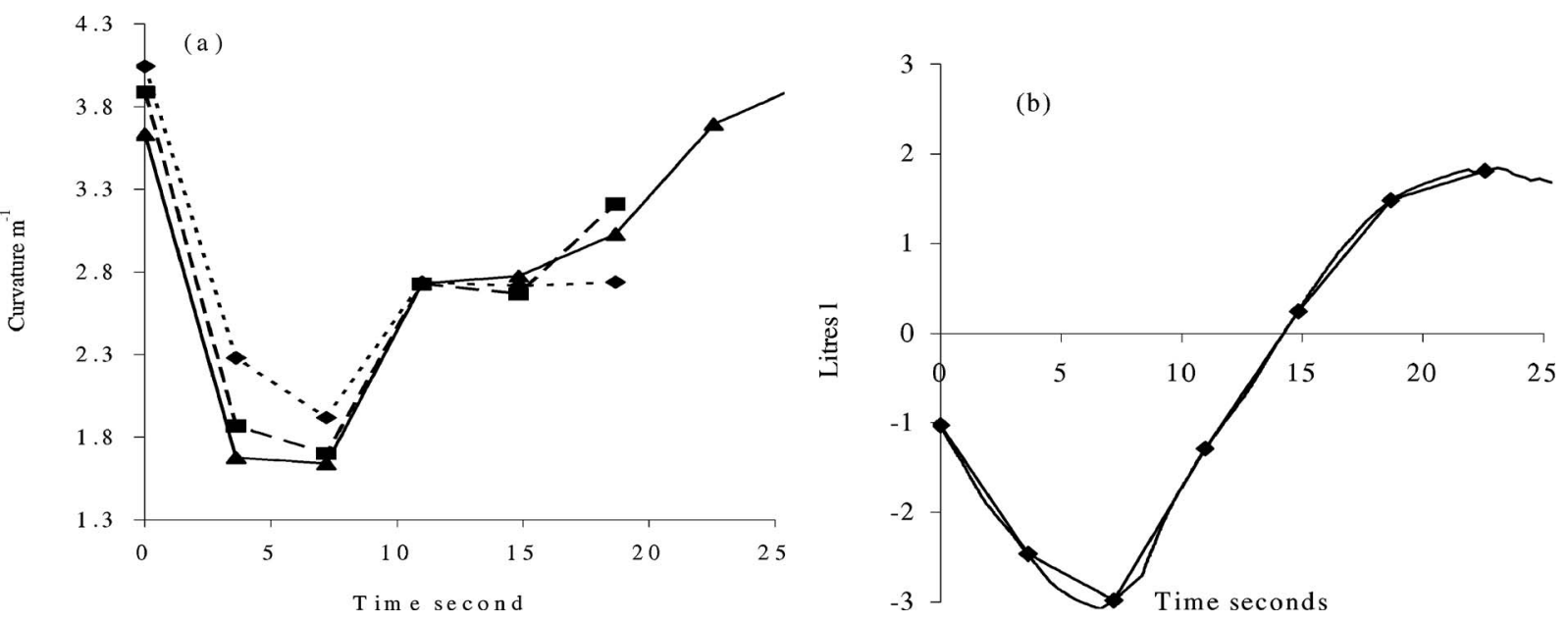

Fig. 11 (a) Monitoring three successive long tidal breaths using a single sensor located on the lower torso. (b) Typical volumetric variation during a long tidal breath, showing a comparison between the measured volume from a spirometer (continuous curve) and a linear fit to the outputs from the four curvature sensors (discrete points). 


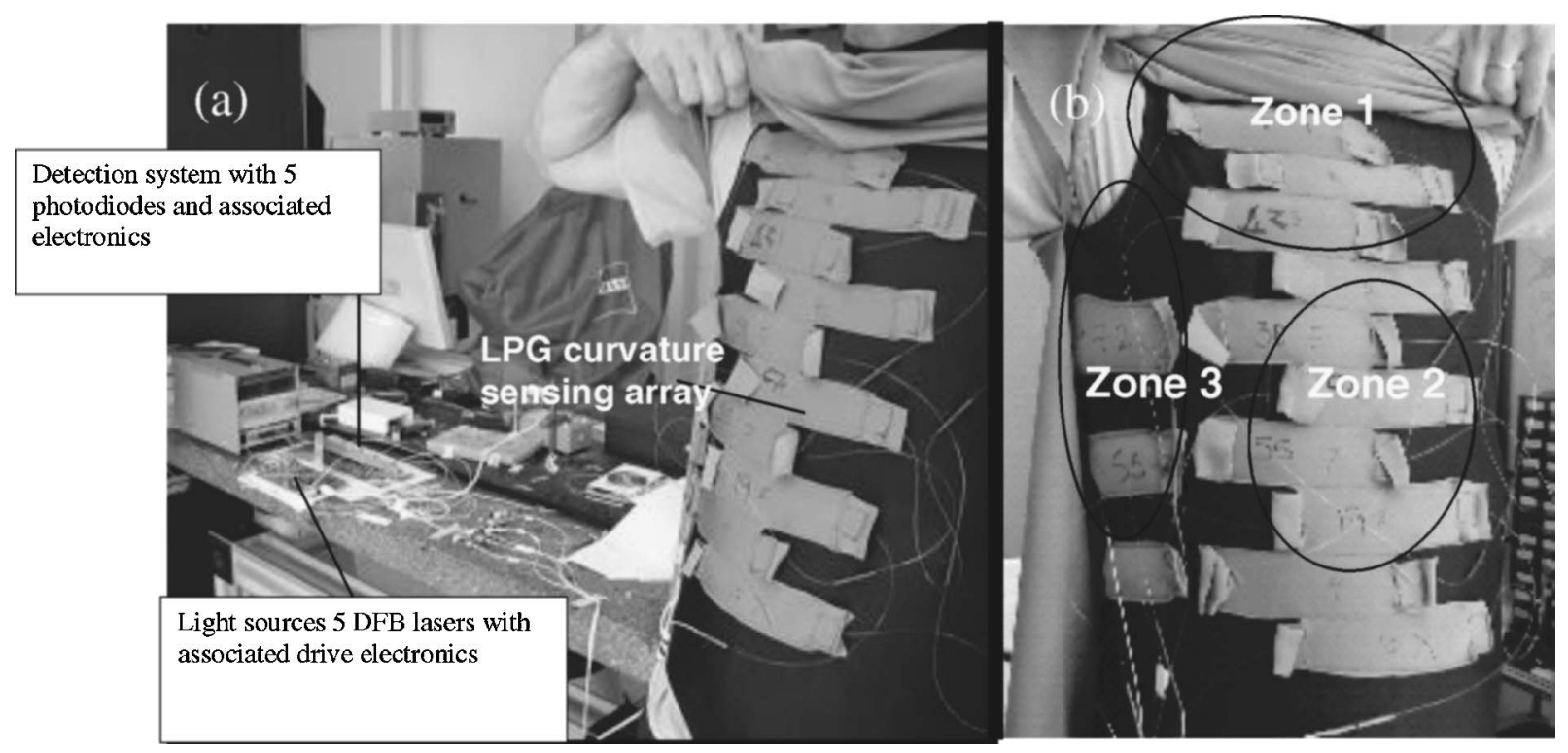

Fig. 12 The curvature sensing scheme for the nine sensors. (a) The full sensing array and the interrogator. (b) Alternative view showing the locations of the sensors on the test subject.

A second, improved sensing garment was also constructed by modifying a commercially available Lycra gymnastic vest, which provided a close contacting surface to the subject's torso. The curvature sensing array consisted of nine sensors, which were interrogated using two DFB lasers [see Figs. 6 and 12(a)]. The sensors were placed in a series of pockets using Velcro, which allowed them to be easily moved; the aim being to choose positions over the torso to enable optimal monitoring of the thoracic and abdominal movements of the human subject during respiration [see Fig. 12(b)].

The sensing array was used to record curvature changes during inspiration and expiration; again the flow being simultaneously recorded at the mouth of the subject with the spirometer used previously. The experiment involved normal rhythmic breathing and transient breathing (rapid exhalation) with subject $\mathrm{X}$ standing and allowed to adopt his own natural posture with his arms at rest by his sides breathing naturally. Several tests (30 trials) were performed on subject $\mathrm{X}$. The duration of each measurement period was approximately one minute, which consisted of typically ten breaths; this was repeated five times to ensure that the sensors measured typical surface motion of the thorax and abdomen.

A typical response to breathing from one of the sensors that is on the monitoring garment is shown for several trials in Fig. 13. While the individual response of the sensor varies from trial to trial, it can be seen that the general behaviors are similar.

After the experiments were completed, linear regression was applied, enabling the curvatures obtained from the nine sensors to predict expired volume. This process assumes that volume $\quad(R)=A \cdot s_{1}(R)+B \cdot s_{2}(R)+C \cdot s_{3}(R)+D \cdot s_{4}(R) \ldots$ $+I \cdot s_{9}(R)$, where the constants $A$ to $I$ are obtained using the SPSS software package, ${ }^{28}$ and $s_{1}$ to $s_{9}$ are the responses of the individual sensors (see Figs. 14-16). The assumption of a linear combination of the curvature sensor outputs was verified by checking that the differences between the predicted values and the observed data had a normal distribution around a mean of zero. In Figs. 14(a) and 14(b), the same linear regression coefficients are used, with the results of Fig. 14(a) used to obtain the coefficients. It can be seen that, while these coefficients reproduce the general variation of volume with time, there seems to an overall constant volume error attached to the data in Fig. 14(b), due to a change in posture of the subject between the trials. These results confirm that the absolute measured volume cannot be inferred from a simple linear combination of the outputs; it will be necessary to use the data to reconstruct the shape of the torso to obtain a true measure of the absolute volume. Work on this aspect will be published elsewhere.

Figure 14(a) shows a close correlation between the measured volume from the spirometer and the volume calculated from the sensing array; also, the change in volume can be measured with just one calibration run of the sensing array, as demonstrated by Fig. 14(b). Second, the results shown in Fig. 15(a) and 15(b) indicate that regional variations of breathing movement on the torso can be detected using the system, i.e., the thoracic and abdominal locations. This shows that this sensing scheme has the potential to measure the total volume

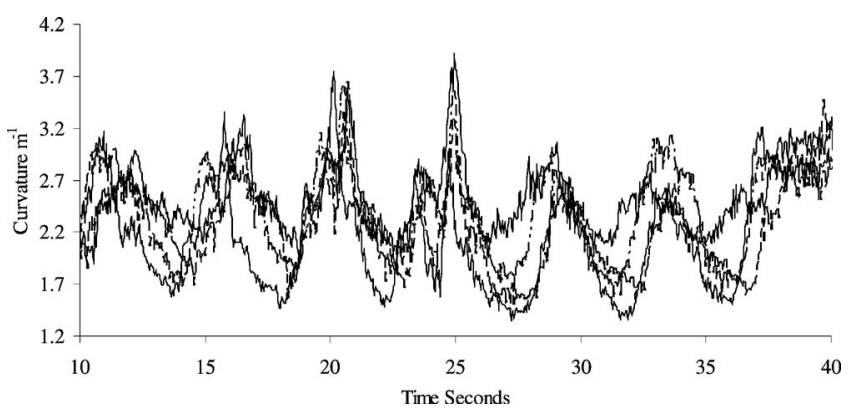

Fig. 13 The measured curvature response of a sensor located at position 1 in zone 1 (the top sensor on the thorax) while the subject is breathing. 

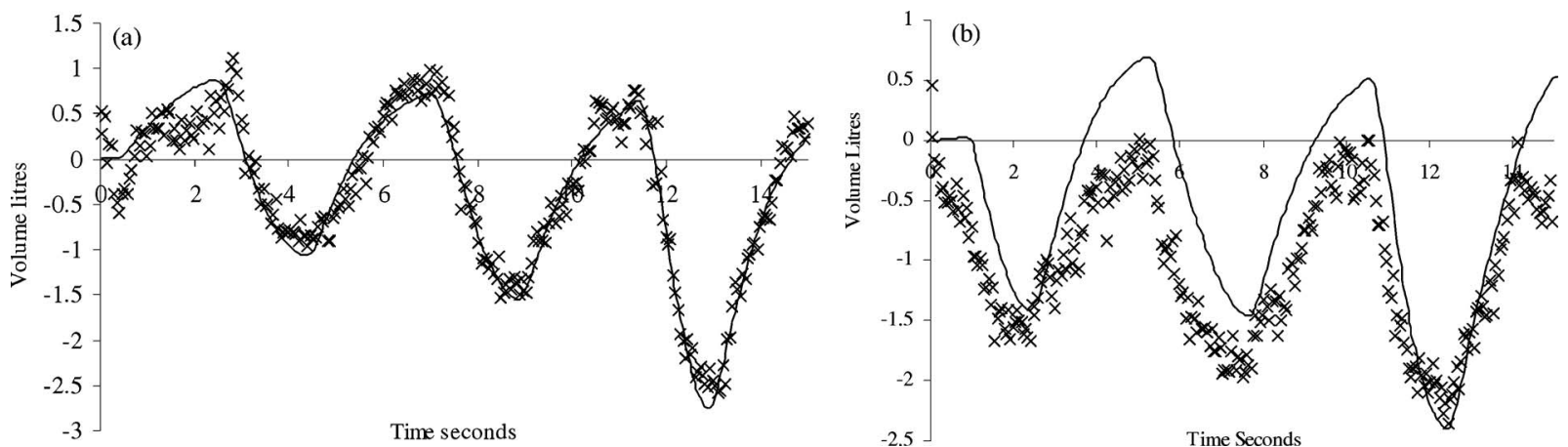

Fig. 14 Typical examples of normal rhythmic breathing showing the measured volume change with time from the spirometer (-) and the predicted volumes for the curvature sensing scheme $(\times)$. Plots (a) and (b) represent different trials using the same linear regression coefficients obtained from the data in (a).

changes and the regional movement that generates those volume changes.

During testing, it was found that three sensors did not produce useful outputs; the positions of these sensors are indicated in Fig. 17(a). The curvature responses of these sensors were closely inspected. Notwithstanding the fact that the curvatures experienced by these sensors seemed to be similar to those of other sensors on the garment and within the designed operational range of the devices, it was found that sensor B showed little response to breathing and the other two sensors $\mathrm{A}$ and $\mathrm{C}$ appeared to be much more noisy than the other six sensors [see Fig. 17(b)]. Preliminary investigations suggest that the location of sensor B simply does not experience much curvature variation. In the case of sensors $A$ and $C$, we suspect that there is a sharp bend causing buckling of the sensor at that point, i.e., a nonuniform curvature, which could significantly distort the shape of the LPGs' attenuation bands.

Using linear regression on all the individual sensors responses, it was found that the volumetric error (one standard deviation) obtained between the curvature sensing array and the measured volume from the spirometer varied from $\sim 12$ to $\sim 6 \%$ of the volume change, as the number of sensors in the array varied from 4 to 9 [see Fig. 18(a)]. It was also observed that data from sensors $\mathrm{A}, \mathrm{B}$, and $\mathrm{C}$, referred to earlier, did not have a significant effect on the volumetric error.

In a similar vein, when looking at the goodness-of-fit parameter shown in Fig. 18(b) (the proportion of variation in the dependent variable explained by the regression model, ranging from 0 to 1 ), sensors $\mathrm{A}, \mathrm{B}$, and $\mathrm{C}$ do not make a significant contribution, while inclusion of the other six sensors causes the goodness of fit to rise to 0.94 .

The investigation reported was done with one subject. To confirm the versatility of the sensing garment, it was also placed on six other subjects with no known respiratory complaints.

All subjects were standing and allowed to adopt their own natural posture with their arms at rest by their sides; all were allowed to breathe naturally, several tests being done with each subject. The duration of each measurement period was approximately one minute or around ten breaths; this was repeated five times to ensure that the sensors measured typical surface motion of the torso. A summary of the results is
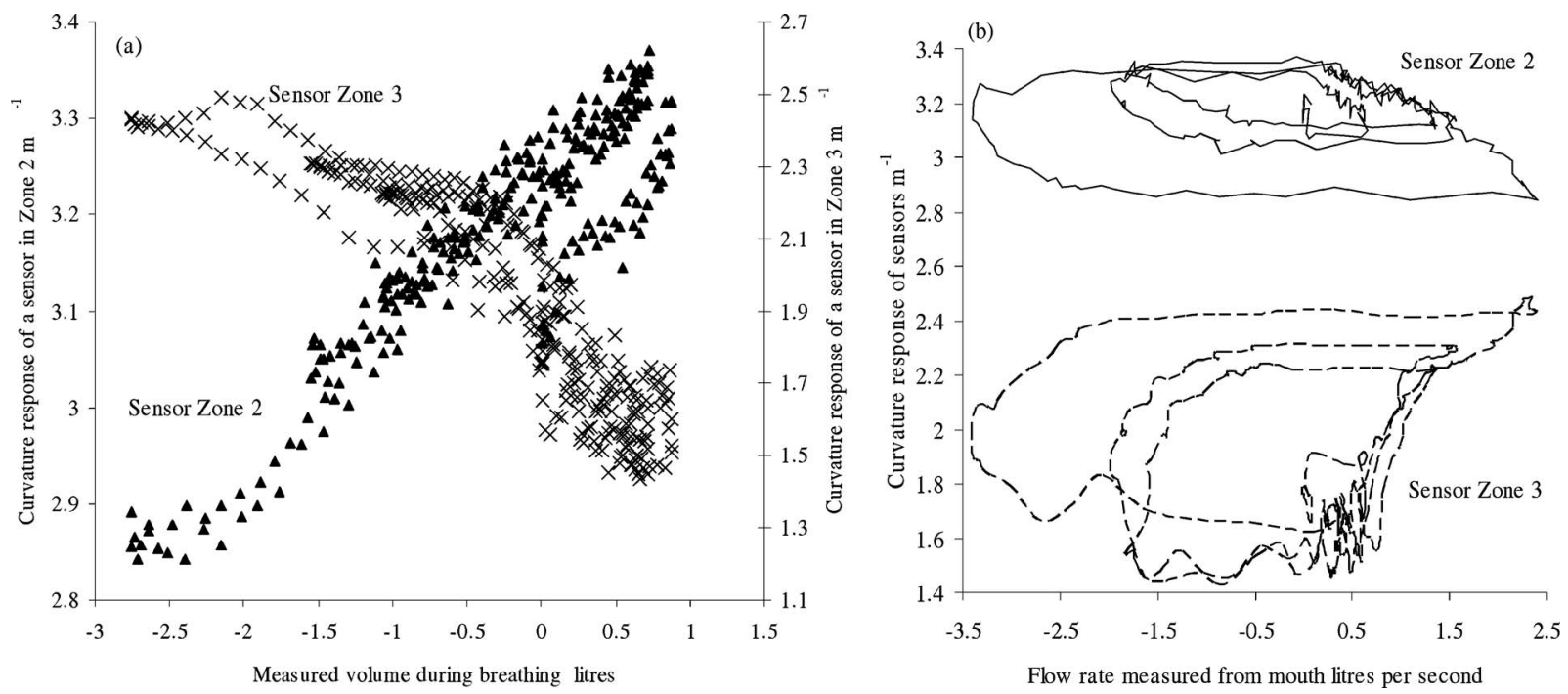

Fig. 15 Typical examples of the regional variation of the detected curvature during respiration over a few breaths: synchronous curvature response of sensors in zone 2 and zone 3 as a function of (a) measured volume at the mouth and (b) the flow rate measured at the mouth. 

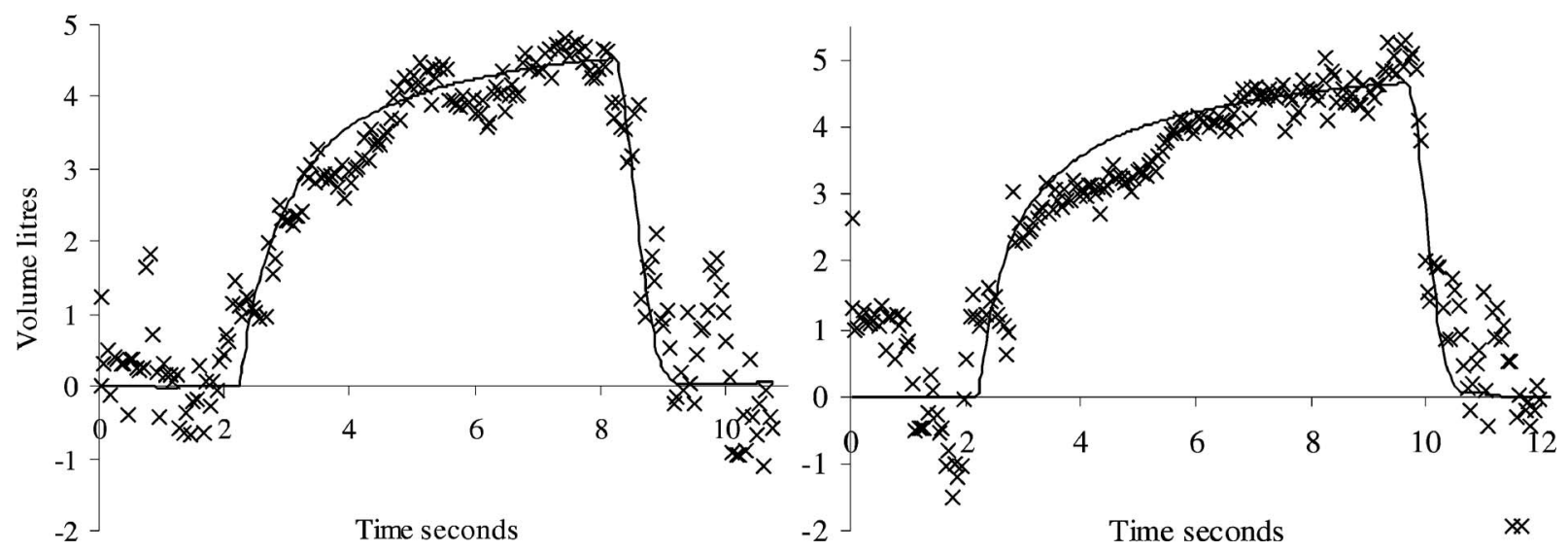

Fig. 16 Two examples of transient breathing (rapid exhalation) showing the measured volume change with time from the spirometer ( -), along with the predicted volumes from the curvature sensing scheme $(\times)$.

shown in Table 1. Additional trials were also conducted with the subjects. First, when the test subjects were asked to breathe deeply, it was found that the failure rate was similar to those shown in Table 1. Second, test subjects were asked to breathe both normally and deeply in a more erect standing posture, and again similar failure rates were found for the sensors on the vest, though these data are not presented in the work to aid brevity.

Subjects were chosen to cover a large variation in body shape, size, and body type (ectomorph, mesomorph, and endomorph) (see Table 1). It was found that the number of sensors that did not function correctly was 0 or 1 for the majority of subjects with just one person (an ectomorph) having three failures (see Table 1). This test seems to suggest that a single sensing garment can be used on a large variety of body types. It can be seen from Table 1 that the sensor failures occurred in all three zones, and that different sensors failed for different subjects. Failure symptoms include curvatures out of the device measurement range and large amounts of noise, perhaps caused by buckling as discussed earlier.
These preliminary trials were conducted on volunteers who were all men. While we have not yet carried out trials with women, the versatility of this approach has been shown. The sensing system will probably need to be adapted to allow for differences in chest shape for women. Sensors above the breasts may reveal important changes in chest movement, but sensors on the under surface of the breast would be less likely to be helpful. These aspects will be tested in subsequent studies. The tests showed a maximum failure rate of three sensors in a nine sensor array (subject A) with a failure occurring in each zone, while for the majority of subjects, only one failure occurred (subjects $\mathrm{C}, \mathrm{D}$, and $\mathrm{E}$ ) with no failures with two subjects (B and $\mathrm{F}$ ).

This is a favorable result when compared to the test subject who was used for the more detailed test and analysis described earlier (subject X). In those trials, it can be seen from Fig. 16 that this test subject had effectively three sensors that failed in a nine sensor array, with a failure occurring in each zone [see Figs. 12(b) and 17(a)]. This appears to be a high failure rate of sensors, but it can be seen from Figs. 18(a) and
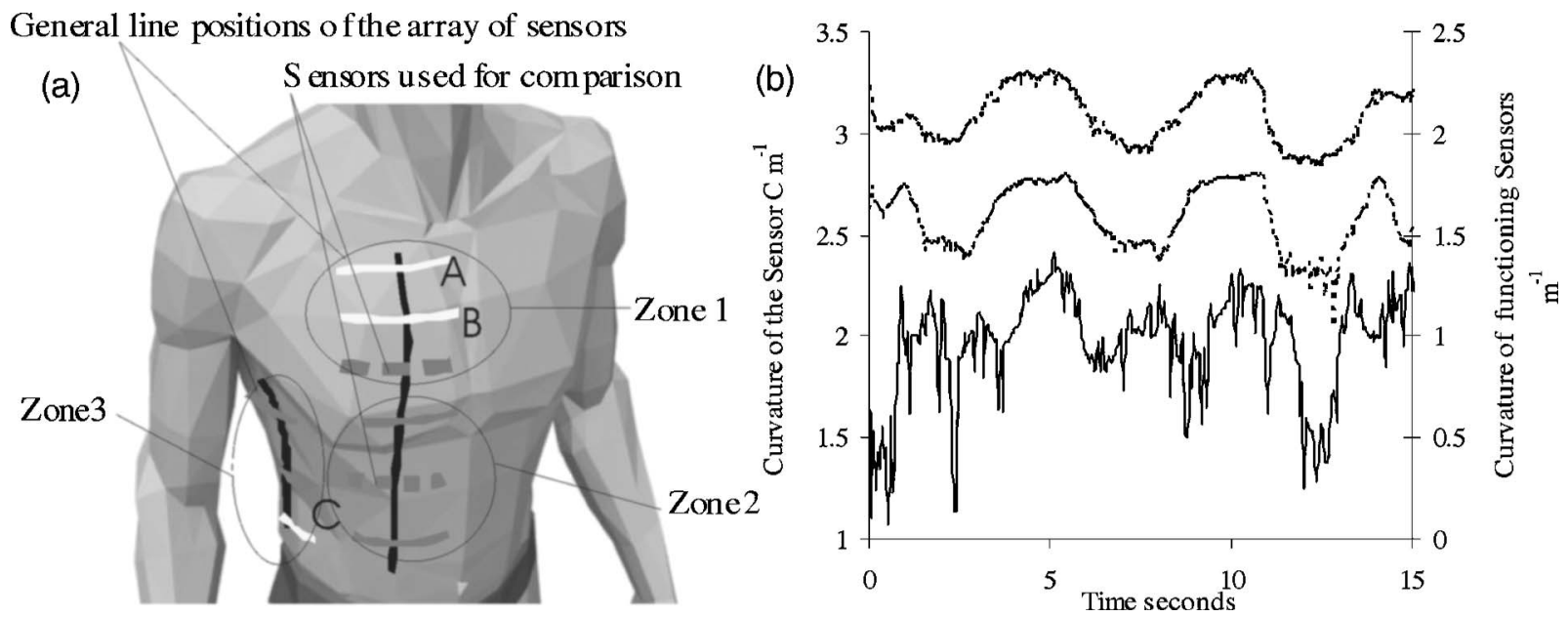

Fig. 17 (a) Schematic of the spatial positions of the curvature sensors on the torso. The white sensors, A, B, and C, are excluded from the linear regression fit to the volume shown in Figs. 14 and 15 (b) Comparison of the performance of two functioning sensors [see Fig. 16(a)] to failed sensor C. 
Table 1 The variety of body types used with the curvature sensing garment.

\begin{tabular}{|c|c|c|c|c|c|c|c|c|}
\hline \multirow[b]{2}{*}{ Subject } & \multirow[b]{2}{*}{$\begin{array}{l}\text { Body } \\
\text { weight } \\
\text { kg }\end{array}$} & \multirow[b]{2}{*}{$\begin{array}{l}\text { Height } \\
\mathrm{cm}\end{array}$} & \multirow[b]{2}{*}{$\begin{array}{l}\text { Chest } \\
\text { circumference } \\
\mathrm{cm}\end{array}$} & \multirow[b]{2}{*}{$\begin{array}{l}\text { Age } \\
\text { Years }\end{array}$} & \multicolumn{3}{|c|}{$\begin{array}{l}\text { Number of } \\
\text { array elements } \\
\text { operational }\end{array}$} & \multirow{2}{*}{$\begin{array}{l}\text { Body } \\
\text { mass } \\
\text { index }\end{array}$} \\
\hline & & & & & $\begin{array}{c}\text { Zone } \\
1\end{array}$ & $\begin{array}{c}\text { Zone } \\
2\end{array}$ & $\begin{array}{c}\text { Zone } \\
3\end{array}$ & \\
\hline A & 60 & 180 & 88.9 & 36 & 2 & 2 & 2 & 18.5 \\
\hline B & 72 & 180 & 96.5 & 45 & 3 & 3 & 3 & 22.5 \\
\hline C & 80 & 177.8 & 104.1 & 65 & 2 & 3 & 3 & 25 \\
\hline D & 82 & 157.5 & 104.1 & 29 & 2 & 3 & 3 & 33.3 \\
\hline$E$ & 98.5 & 182.9 & 108 & 29 & 3 & 2 & 3 & 29.4 \\
\hline $\mathrm{F}$ & 105 & 195.6 & 115 & 46 & 3 & 3 & 3 & 29.1 \\
\hline
\end{tabular}

18(b) that even excluding these sensors, we obtained a volumetric error of $\sim 6 \%$ with a 0.94 goodness of fit. Comparing subject X's test results with the larger test group used, subject A had three failures, a sensor in each zone, while the rest of the test group had fewer failures with no multiple failures in each zone (see Table 1). Again, sharp bending or buckling of the sensors is likely to be responsible for the failures. Also, it can be seen from the test that zone 1 yields the greatest number of failures overall. The failures in this zone are probably due to the relative flatness of this area and the associated small movement of this part of the thorax during breathing (i.e., the sternum for some subjects). This suggests that the sensor curvature sensitivity may need to be increased for this position on the torso. Also inspecting Table 1, excluding subject A who has a low-body mass index, in zone 2 there is only one failure, for subject $\mathrm{E}$, which may be due to the fitting of the garement on the subject, and there are no failures in zone 3. Furthermore, sensor failure rate and location does not appear to be correlated with the body mass index of the subjects.

While the authors understand that this is a small test group, it can be seen that there is a large diversity in the shape of the subjects, i.e., weight, height, chest circumference, and body mass index, making this group an appropriate sample to test the sensing array's versatility.

Comparing the performance of our system against those of other researchers based on magnetometry and inductive sensors (such as Refs. 29 and 30) is quite hard. This is due to the fact that data from the latter systems are used in conjunction with chest models constructed by Konno et al. ${ }^{1}$ and Smith et al., ${ }^{31}$ while in our case no model is assumed due to the fact that our system provides direct detection of the variations in the shape of the torso during breathing.

Concerning the procedure to obtain the volume change during breathing: in our scheme, the sensors are initially calibrated with respect to curvature in the laboratory. At the moment, calibration against a spirometer is also required for each subject; however, current development of our sensor technology is aimed at using the curvature data to reconstruct the shape of the thorax and abdomen, allowing absolute volumetric data to be obtained without calibration (to be reported at a later date), which is not the case in the schemes described in Refs. 29 and 30.
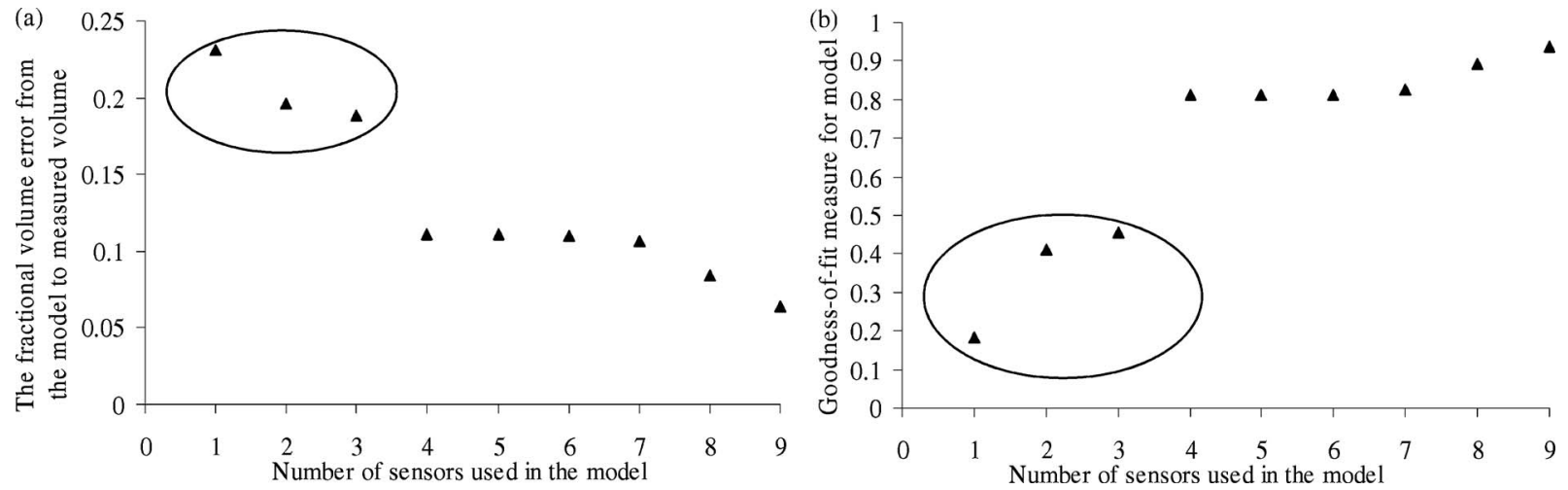

Fig. 18 The performance of the curvature sensing scheme referenced to a spirometer: (a) the volumetric error from the sensing array referenced to the measured volume obtained using the spirometer as a function of the number of sensors. (b) The goodness of fit of the linear regression model as a function of the number of sensors. The circled data refer to the "failed" sensors. 
The physical size and weight of our detection scheme can be separated into two parts. The interrogation unit is approximately $20 \times 15 \times 8 \mathrm{~cm}$ with a weight of $1 \mathrm{~kg}$. Second, the sensing garment is lightweight and has little bulk. The size of this interrogation system is comparable to other systems that have been reported. ${ }^{29,30}$ While the authors acknowledge that this prototype is too bulky to be considered mobile, it is true to say that the electronic and optical components of the interrogation scheme are based on telecommunications technology, which is relatively inexpensive and small, and consequently the interrogation unit has the potential to be reduced considerably in size and weight. Thus we strongly believe that this system has a real potential to be made portable.

The sensing garment reported here has nine sensors over the thoracic and abdominal regions of the torso (extendable to 20 with the current interrogation system), which directly yield regional variation in shape change during breathing (see Fig. 15). This is an advantage over the existing magnetometry and inductive sensor systems, such as described in Refs. 29 and 30 , which can only detect motion in three locations on the torso. While in our case the test group is small and extensive analysis is done with only one subject, the volumetric error obtained with six functioning sensors compared to a spirometer was just $6 \%$ over a series of trials. Taken in conjunction with the results in Fig. 17 and the data shown in Table 1, this compares well against reported studies of other portable systems, which yield errors of $\sim 10 \%$ (Ref. 29 ) and $\sim 16 \%$ (Ref. $30)$.

The authors realize that the major disadvantage of the present sensing system is that it does not measure absolute volume changes. This is not an intrinsic problem with the approach; in fact, a new sensor array configuration is being implemented to overcome this problem by providing true shape reconstruction.

\section{Conclusion}

As far as we are aware, we report on the first practical interrogation and multiplexing scheme to be demonstrated with LPG sensors. An array of up to nine long-period-gratingbased curvature sensors on a garment are used to monitor the thoracic and abdominal movements of a human during respiration. These results are used to obtain volumetric tidal changes of the human torso, which show agreement with a spirometer used simultaneously to record the inspired and expired volume at the mouth, with a volumetric error of $6 \%$ obtained with just six sensors. The sensing garment is also used on a group of six human males with differing body dimensions and with marked variation in body shape. The garment functioned with an average sensor failure rate of $\sim 8 \%$.

Respiratory medical practice has many potential applications for a vest that can sense thoracic and abdominal movement, and from this derive an accurate output of ventilation with respect to time. Any method that requires a mouthpiece for the subject to breathe through alters the resting ventilatory state and so potentially corrupts the diagnostic accuracy of the technique. Currently, inductance plethysmography allows a semiquantitative estimate of ventilation without the use of a mouthpiece, ${ }^{5}$ but it has a limited ability to define regional ventilation within the thorax. An optical system using 80 sensor points on the thorax has been developed, but restricts the subject to having the chest bare and remaining in one location relative to four computerized cameras, ${ }^{26}$ and is therefore not suitable for ambulatory monitoring.

There are areas of work that still need to be done in terms of the effects of posture, in that all the studies reported here are in subjects standing upright. The effect on ventilation sensed on the surface by our device and at the mouth while a subject changes from supine to standing and to seated needs exploring. The future applicability of this device may require further technological advances with fibers being woven into conventionally designed garments. This would allow sensors to be optimally placed to take into account the contour differences between individuals and between the sexes. Furthermore, this approach would be much more acceptable to wear than externally applied plethysmographic jackets. ${ }^{30}$ Our interrogation system based on derivative spectroscopy has the potential to be made small and portable, thus enabling unobtrusive ambulatory monitoring of breathing.

\section{Acknowledgment}

The authors would like to thank Aerovac Systems Limited of Keighley, West Yorkshire, United Kingdom, for their help in the development of the sensors, with special thanks to Matthew Fisher. We would also like to thank Tim Hart of Fibercore Limited for fruitful discussions and for supplying the material constants and specification of the PTL fiber. This work was funded by the UK Engineering and Physical Sciences Research Council and the Wellcome Trust under project 074649/Z/04/Z.

\section{References}

1. K. Konno and J. Mead, "Measurement of the separate volume changes of the rib cage and abdomen during breathing," J. Appl. Physiol. 22(3), 407-422 (1967).

2. H. Watson, "The technology of respiratory inductance plethysmography," ISAM Proc. 3rd Intl. Symp. Ambulatory Monitoring, p. 537, Academic, San Diego, CA (1980).

3. S. J. Cala, C. M. Kenyon, G. Ferigno, P. Carnevali, A. Aliverti, P. T. Maklem, and D. F. Rochester, "Chest wall and lung volume estimation by optical reflectance motion analysis," J. Appl. Physiol. 81(6), 2680-2689 (1996).

4. S. Levine, D. Silage, D. Henson, J. Wang, J. Kreig, J. LaManca, and S. Levy, "Use of triaxial magnetometer for respiratory measurements," J. Appl. Physiol. 70(5), 2311-2321 (1991).

5. R. B. Banzett, S. T. Mahan, D. M. Garner, A. Brughera, and S. H. Loring, "A simple reliable method to calibrate magnetometers and respitrace," J. Appl. Physiol. 79(6), 2169-2176 (1995).

6. T. Earthrowl-Gould, B. Jones, and M. R. Miller, "Chest and abdominal surface motion measurement for continuous monitoring of respiratory function," Proc. Inst. Mech. Eng., Part H: J. Eng. Med. 215(5), 515-520 (2001).

7. T. Allsopa, T. Earthrowl-Gould, D. J. Webb, and I. Bennion, "Embedded progressive-three-layered fiber long-period gratings for respiratory monitoring," J. Biomed. Opt. 8(3), 552-558 (2003).

8. T. Allsop, A. Gillooly, V. Mezentsev, T. Earthgrowl-Gould, R. Neal, D. J. Webb, and I. Bennion, "Bending and orientational characteristics of long period gratings written in D-shaped optical fiber," $J$. Lightwave Technol. IEEE Trans. Instrum. Measurement 53(1), 130135 (2004).

9. T. Allsop, D. Webb, and I. Bennion, "A comparison and characteristics of sensing parameters of long period gratings written in three different types of fiber," Opt. Fiber Technol. 9(4), 210-223 (2003).

10. V. Bhatia, "Applications of long-period gratings to single and multiparameter sensing," Opt. Express 4(11), 457-466 (1999).

11. T. Allsop, T. Earthrowl-Gould, R. Reeves, D. J. Webb, and I. Bennion, "The interrogation and multiplexing of long period gratings curvature sensors using derivative spectroscopy technique based upon 
fiber Bragg gratings," Meas. Sci. Technol. 15(1), 44-48 (2004).

12. H. J. Patrick, A. D. Kersey, and F. Bucholtz, "Analysis of the response of long period fiber gratings to external index of refraction," J. Lightwave Technol. 16(9), 1606-1612 (1998).

13. H. J. Patrick, C. C. Chang, and S. T. Vohra, "Long period fiber gratings for structural bend sensing," Electron. Lett. 34(18), 1773-1775 (1998).

14. V. Bhatia, "Properties and sensing applications of long-period gratings," PhD Thesis, Virgina Polytechnic Institute and State University, Blackburg, Virgina (1996).

15. M. Heiblum and J. H. Harris, "Analysis of curved optical waveguides by conformal transformation," IEEE J. Quantum Electron. QE-11(2), 75-83 (1975).

16. L. Zhang et al., "Design and realization of long-period grating devices in conventional and high birefringence fibers and their novel applications as fiber-optic load sensors," IEEE J. Sel. Top. Quantum Electron. 5(5), 1373-1378 (1999).

17. T. Miyanaga et al., "Simplified human body-model for evaluating thermal radiant environment in a radiant cooled space," Build. Environ. 36(7), 801-808 (2001).

18. P. Kluczynski, "Wavelength modulation absorption spectrometry-an extensive scrutiny of the generation of signals," Spectrochim. Acta, Part B 56(8), 1277-1354 (2001).

19. T. Allsop, R. Reeves, R. Neal, and I. Bennion, "A high accuracy/ sensitive refractive index sensing system based upon a long period grating Mach-Zehnder interferometer with a heterodyne detection technique," Rev. Sci. Instrum. 73(4), 1702-1705 (2002).
20. W. Du, H. Tam, M. Liu, and X. Tao, "Long-period fiber grating bending sensors in laminated composite structures," Proc. SPIE 3330, 284-292 (1998).

21. H. Patrick G. Williams, A. Kersey, J. Pedrazzani, and A. Vengsarkar, "Hybrid fiber Bragg grating/long period fiber grating sensor for strain/temperature discrimination," IEEE Photonics Technol. Lett. 8(9), 1223-1225 (1998).

22. J. Ma et al., "Optical-fiber sensor for simultaneous measurement of pressure and temperature: analysis of cross sensitivity," Appl. Opt. 35(25), 5206-5210 (1996).

23. X. Shu et al., "Room-temperature operation of widely tunable loss filter," Electron. Lett. 37(4), 216-218 (2001)

24. C. Tsao, Optical Fibre Waveguide Analysis, Oxford, New York (1992).

25. Little Anne ${ }^{\mathrm{TM}}$ CPR Manikin, Laerdal Medical, see http:// www.laerdal.co.uk/.

26. SolidWorks ${ }^{\mathrm{TM}}$, see http://www.solidworks.com/.

27. A. Alverti et al., "Optoelectronic plethysmography in intensive care patients," Am. J. Respir. Crit. Care Med. 161, 1546-1552 (2000).

28. SPSS 12.0 .1 for Windows ${ }^{\mathrm{TM}}$, see http://www.spss.com/.

29. F. D. McCool et al., "Tidal volume and respiratory timing derived from a portable ventilation monitor," Chest 122, 684-691 (2002).

30. C. F. Clarenbach, O. Senn, T. Brack, M. Kohler, and K. E. Bloch, "Monitoring of ventilation during exercise by a portable respiratory inductive plethysmograph," Chest 128, 1282-1290 (2005).

31. R. Smith et al., "Three degree of freedom description of movement of the human chest wall," J. Appl. Physiol. 60, 928-934 (1986). 